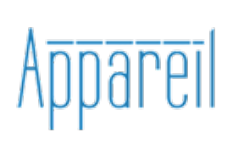

Appareil

$17 \mid 2016$

Art et médium 1 : le médium de l'art

\title{
Kant, les beaux-arts et leurs moyens d'expression
}

\section{Danielle Lories}

\section{(2) OpenEdition}

Journals

Édition électronique

URL : http://journals.openedition.org/appareil/2282

DOI : 10.4000/appareil.2282

ISSN : 2101-0714

Éditeur

MSH Paris Nord

\section{Référence électronique}

Danielle Lories, « Kant, les beaux-arts et leurs moyens d'expression», Appareil [En ligne], 17| 2016, mis en ligne le 11 juillet 2016, consulté le 30 juillet 2020. URL : http://journals.openedition.org/appareil/ 2282 ; DOI : https://doi.org/10.4000/appareil.2282

Ce document a été généré automatiquement le 30 juillet 2020.

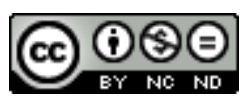

Appareil est mis à disposition selon les termes de la Licence Creative Commons Attribution - Pas d'Utilisation Commerciale - Pas de Modification 4.0 International. 


\title{
Kant, les beaux-arts et leurs moyens d'expression
}

\author{
Danielle Lories
}

\section{Introduction}

1 Afin d'éclairer la notion de médium artistique apparue dans les théories de l'art au cours du $\mathrm{xx}^{\mathrm{e}}$ siècle, on se propose ici de remonter le fil du temps bien en deçà de l'art moderne. On est en effet en droit de penser que la question du médium « est peut-être une résurgence d'un problème plus ancien des disciplines artistiques, autrement dit de l'idée même d'un pluriel, les arts, qui viendrait compartimenter la grande idée de l'Art, au singulier ${ }^{1} »$, mais on pourrait aussi bien dire que la question de l'unité des disciplines que nous considérons aujourd'hui comme des « compartiments» de l'Art fut bien plus longue à s'imposer que la question du «compartimentage» de l'Art ${ }^{2}$. En effet, la question du principe de rassemblement des arts était loin d'être aisée à trancher dans l'héritage non seulement de la pluralité des Muses, mais aussi des divisions médiévales entre les arts libéraux (dignes de l'homme libre) et les arts "mécaniques » ou «mercenaires » (exercés par les serfs ou contre rémunération). Aucun de ces groupes ne fournit l'ancêtre de la liste des «beaux-arts » qui s'établit au xvIII ${ }^{\mathrm{e}}$ siècle. Le pluriel des beaux-arts précède l'usage d'un singulier qui les rassemblera (tout en admettant une hiérarchie et une articulation des différences) et dont nous sommes encore tributaires dans notre usage actuel, lorsque nous parlons de « subdiviser » l'Art (ou l'art).

2 Le présent propos sera centré sur une approche de ce que l'on peut appeler les médiums artistiques, si l'on entend par là que "d'abord matière, le médium est la transcendance d'un matériau d'origine et son exploitation artistique ${ }^{3}$. » Il sera question de la classification des beaux-arts (et accessoirement de la hiérarchie qui en ressort) proposée par Kant, à la charnière, très signifiante à cet égard, des XVIII et XIX ${ }^{\mathrm{e}}$ siècles. Comme le siècle près de s'achever, Kant parle des "beaux-arts", s'interroge sur la manière de les classer, de distinguer et lier une pluralité unifiée sous cette appellation commune, mais n'évoque pas l'«Art »: le siècle suivant le fera. Pourtant le principe de 
l'unité des beaux-arts n'est plus celui, typique encore du XviII ${ }^{\mathrm{e}}$ siècle, de l'imitation de la belle nature. Et il n'est pas encore cette essence unique de l'Art au singulier qui, chez Hegel, se déclinera dans l'enchaînement systématique, historique mais surtout logiquement nécessaire des formes d'art et des arts particuliers qui ont en commun de se destiner au beau, c'est-à-dire à la manifestation sensible de l'Idée.

\section{Kant et ses références}

Dans la "Critique de la faculté de juger esthétique ", au moment où Kant aborde la «Division des beaux-arts", la beauté est définie comme "l'expression d'idées

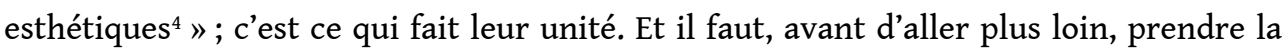
mesure de la distance prise par rapport aux critères d'unité qu'avait reconnus son siècle et que ses contemporains (pré-)romantiques n'avaient de cesse de contester.

Pour ce regard rétrospectif inévitable, Kant nous offre un point de départ car sa troisième Critique n'évoque que deux théoriciens qu'il qualifie de "critiques du goût »: Batteux et Lessing 5 . Tous deux sont des repères tout à fait essentiels pour la question de l'unité et de la pluralité des arts au XVIII ${ }^{\mathrm{e}}$ siècle.

5 Dans son essai incontournable sur l'histoire du système des beaux-arts ${ }^{6}$, P. O. Kristeller relève l'« origine relativement récente » de ce système qui n'a pas "adopté sa forme définitive avant le XVIII ${ }^{e}$ siècle ". L'historien fait jouer un rôle clé à l'ouvrage de Charles Batteux, Les Beaux-Arts réduits à un même principe $(1746)^{7}$, selon lequel les arts seraient beaux d'imiter la belle nature ${ }^{8}$.

6 Batteux éprouve encore le besoin de distinguer les beaux-arts des arts mécaniques en donnant aux premiers pour fin le plaisir, de sorte qu'une tripartition des arts selon leur fin s'établit: les arts mécaniques doivent satisfaire le besoin; les beaux-arts visent le plaisir ${ }^{9}$; enfin, sorte d'intermédiaire entre les deux premières, une espèce d'arts a «pour objet l'utilité et l'agrément tout à la fois: tels [sic] sont l'éloquence et l'architecture ${ }^{10} »$.

7 Après l'«étape décisive vers un système des beaux-arts » que constitue la thèse de Batteux, « la touche finale » audit système serait apportée par les Encyclopédistes ${ }^{11}$. En effet, le «Discours préliminaire » de d'Alembert consacre la liste des cinq beaux-arts que sont la peinture, la sculpture, l'architecture, la poésie et la musique : ce sont des arts voués au plaisir et à l'imitation. Par rapport aux divisions de Batteux, l'architecture rejoint plus pleinement le statut de bel-art, tandis que l'éloquence et la danse disparaissent de la liste des beaux-arts "par excellence $»^{12}$. L'Encyclopédie contribua sans nul doute à populariser sous cette forme le système des beaux-arts en Europe, et les cinq arts indiqués comme majeurs par Kristeller - architecture, sculpture, peinture, musique et poésie - sont ceux retenus par l'Esthétique de Hegel quand il s'agit de proclamer l'insuffisance, en matière de jugement sur les arts, des critères, typiques du siècle précédent, du plaisir du goût et de l'imitation.

8 Il faut d'abord constater qu'il ne reste presque rien du classique principe d'imitation chez Kant, puisque la notion même d'imitation est marginalisée à l'extrême. Si elle revêt une certaine portée du point de vue critique, c'est en un sens fort différent de ce qui se trouvait encore chez un Batteux. Ce qu'écrit Kant sur ce point doit être justement interprété : « La nature était belle lorsqu'en même temps elle avait l'apparence de l'art ; et l'art ne peut être appelé beau que si nous sommes conscients qu'il s'agit d'art et que 
celui-ci prend cependant pour nous l'apparence de la nature $\mathrm{e}^{13} »$. Ce que la thèse peut avoir de commun avec celle du principe unique de Batteux, c'est de renvoyer au goût lui-même ${ }^{14}$, au jugement de goût (dans sa pureté) chez Kant. Car la justification de ce parallèle entre nature et art est bien à trouver dans l'appel à la définition selon laquelle « est beau ce qui plaît dans le simple jugement appréciatif (et non pas dans la sensation des sens, ni par l'intermédiaire d'un concept $\left.{ }^{15}\right)$ ». Cette manière de présenter l'appréciation du goût indique, dès à présent, que la différenciation des arts du beau chez Kant ne se fera pas prioritairement par la distinction des sens auxquels ils s'adressent, comme c'était le cas chez Batteux. Quel est donc le sens kantien du parallèle ? C'est la nature qui se montre à nous comme artiste dans la mesure où elle apparaît (et l'on ne peut pas du tout s'assurer qu'il y a plus qu'une simple apparence !) comme produisant "intentionnellement» (comme un peintre son tableau) certaines formes que nous jugeons belles, comme si elle les produisait précisément à cette seule fin de nous plaire. Quant au génie créateur des beaux-arts, on peut sur cette base dire qu'il imite la nature en produisant à son tour des formes susceptibles de nous plaire en suscitant le jeu harmonieux et libre de nos facultés cognitives. Mais en aucun cas il n'est pour cela question que ces formes qui relèvent de l'originalité du génie imitent des formes de la nature, fût-ce de la belle nature. Cela n'est nullement requis. Si la nature se donne à voir comme si elle était portée par des fins, l'œuvre des beaux-arts, portée bien sûr par la poursuite consciente et volontaire de fins par l'artiste, ne sera belle que si cette finalité se fait aussi discrète que possible, aussi discrète qu'un semblant («comme si ») de fin de la nature. Telle est l'imitation de la nature au sens kantien.

9 L'essentiel est ici, du point de vue du débat du xvIII siècle, la condamnation de la «manière » et de l'artifice académique: toute trace du travail scolaire, pénible et soumis à des règles doit s'effacer. Mais cet effacement n'est surtout pas destiné à tromper l'œil ni à faire croire à un ouvrage de la nature: que l'art doit avoir l'apparence de la nature signifie qu'il doit sembler aussi « naturel » et spontané qu'une production de la nature; on ne doit y voir ni témoignage de contrainte ou de soumission, ni trace d'artifice. L'illusion est écartée, le texte est explicite: «il faut prendre conscience qu'il s'agit d'art et non pas de nature ». Ce qu'il s'agit d'effacer n'est pas même "l'accord avec des règles ", c'est au contraire l'exactitude dans cet accord qui donne à l'art l'apparence de la nature, car les productions de la nature elles-mêmes paraissent produites selon des règles fermes et constantes. Ce qu'il faut effacer, c'est l'accord laborieux et la règle énonçable, connue, et reconnue, c'est la "forme scolaire ", c'est-à-dire " une trace manifestant que la règle était présente sous les yeux de l'artiste et qu'elle a imposé des chaînes aux facultés de son esprit ${ }^{16} »$. Cette exclusion de la servilité à l'égard d'un modèle ou d'une règle préalable se répète du reste à l'égard des devanciers, des génies des générations antérieures qui pourtant peuvent servir de guides $^{17}$.

10 Si la mise en ordre de Batteux est tributaire d'une tradition déjà longue qui accompagne l'émergence de la notion de beaux-arts et contribue à la rendre possible tout en l'orientant, celle de l'ut pictura poesis, on sait quel rôle critique joua Lessing à cet égard, en particulier avec le Laocoon ou des frontières de la peinture et de la poésie (1766) ${ }^{18}$. La nouveauté de cet essai ne consiste pas à opposer, dans des termes alors déjà bien connus, la simultanéité caractéristique de la peinture (plus largement des arts plastiques) comme art se donnant pour objet des corps dans l'espace et la temporalité inhérente à la poésie, qui a pour objet des actions se succédant dans le temps. Elle 
consiste bien plutôt dans la charge menée, sur la base de ces différences, contre l'ut pictura poesis. Ce faisant, le texte présente plusieurs caractéristiques qui méritent ici d'être relevées. D'une part, le portrait du critique (littéraire ou d'art) qu'esquisse et défend Lessing prend le contre-pied d'une critique prétendant s'appuyer sur des règles a priori et intangibles, aussi bien que celui du projet récent d'une esthétique comme science $^{19}$. Le critique de Lessing possède des traits que l'on retrouve chez le spectateur du beau chez Kant, auteur d'un jugement libre et désintéressé, subjectif mais à visée universelle, un jugement personnel qui a droit de cité, qui est public, selon une exigence kantienne caractéristique de sa compréhension de l'Aufklärung. Et de même que la faculté d'imagination est au cœur de ce jugement du critique ou du spectateur chez Lessing, de même c'est l'imagination (Einbildungskraft) qui est au cœur et du jugement (du goût) et du génie kantiens, et les conceptions de cette faculté sont parente ${ }^{20}$. Et Kant récuse également la « science » esthétique de Baumgarten : « Il n'y a pas de science du beau, mais il n'en existe qu'une critique, et il n'y a pas de belle science, mais seulement des beaux-arts ${ }^{21}$." En outre, on peut dire qu'en portant l'accent sur les différences entre les arts de l'espace et les arts du temps, en insistant sur les limites de la peinture (vouée à la représentation des corps) par rapport à la poésie (portant sur les actions), Lessing fait pencher la balance du sens signifié du côté d'une valorisation de la poésie plus marquée encore que chez les classiques. Mais cette valorisation est, cette fois, celle de l'expression du sens comprise de telle sorte qu'elle ne peut que renvoyer à l'usage de la parole, et tend ainsi à conforter une hiérarchie affirmant la supériorité incontestée de la poésie sur tous les arts des figures (dans l'espace ${ }^{22}$. La suite montrera en quoi le modèle de l'expression verbale est au cœur du classement kantien des arts.

11 En termes d'attention accordée au propre de chaque art, on ne peut douter que Kant soit à situer plus près de Lessing que de Batteux. Chez celui-ci, l'ut pictura poesis est à l'œuvre au point qu'il affirme qu'il n'est pas besoin de redire de la peinture (et cela veut dire des arts plastiques en général) ce qu'on a dit de la poésie : « ces deux arts ont entre eux une si grande conformité, qu'il ne s'agit, pour les avoir traités tous deux à la fois que de changer les noms, et de mettre peinture, dessin, coloris, à la place de poésie, de fable, de versification.» Un mot donc suffit sur les «moyens dont se sert la peinture pour imiter et exprimer la nature ", pourvu qu'on ait déjà tout dit de la poésie ${ }^{23}$.

\section{L'expression et les idées esthétiques}

Plutôt qu'au nom de l'imitation de la belle nature chez Batteux, ou de l'imitation ou expression de la beauté des corps visibles par la peinture, des actions par la poésie chez Lessing, Kant rassemble les arts du beau en tant que chacun d'eux exprime des idées esthétiques. Cette notion est dès lors capitale pour comprendre sa manière de classer et de hiérarchiser les arts ${ }^{24}$.

Le génie investit le champ de la pensée non pas avec des images, mais avec des «idées esthétiques ». Le génie, c'est l'imagination créatrice dans toute sa liberté, ce qui ne veut pas dire l'arbitraire de la fantaisie débridée (ni du rêve, ni du phantasme absurde). Celle-ci serait incommunicable, ce qui est interdit par la norme du goût (l'universalité subjective) dans les limites de laquelle le génie doit se tenir ${ }^{25}$. C'est du champ de la pensée qu'il est ici question, et non du délire irrémédiablement privé. Ce qui revient à dire que les bornes sont celles du sens. 

clair que c'est elle qui mène le jeu, un jeu qui mêle subtilement imagination, entendement (dépassé) et raison. Elle est toute spontanée. La limite inhérente à l'imagination est qu'elle peut présenter un concept d'entendement, à des fins de connaissance, mais elle ne peut présenter de la même façon une idée de la raison, qui demeure dès lors un objet de pensée mais jamais de connaissance. Pourtant, dans sa créativité, le génie lève autant que faire se peut cette limite. La description de l'esprit génial indique en effet comment l'imagination surmonte, par voie détournée : indirecte et symbolique, son impuissance par rapport à la raison ${ }^{26}$. L'enjeu est l'impossibilité irrémédiable d'une présentation objective (qui en permettrait une connaissance, une science) du suprasensible. L'imagination se fait suggestive, indicatrice, elle fait signe vers le champ illimité du sens (non de la « vérité » de la science), et ce dans les limites de la finitude. Dans l'impossibilité de procurer à l'idée son image, comme elle le fait pour un concept empirique, l'imagination n'est pas dépourvue de tout pouvoir à l'égard de ce qui se donne à penser seulement, mais son activité doit être conçue comme de signification : il s'agit de faire signe vers l'objet de pensée. Que l'imagination comme faculté des idées esthétiques ouvre le champ de la pensée, le texte le dit à de multiples reprises de façon expresse :

Par idée esthétique, j'entends cette représentation de l'imagination qui donne beaucoup à penser, sans pourtant que toutefois aucune pensée déterminée, c'est-àdire aucun concept, ne puisse lui être adéquate, et que par conséquent aucun langage (Sprache) n'atteint complètement ni ne peut rendre compréhensible ${ }^{27}$.

Aucun mot (concept) ne l'atteint, n'en rend compte totalement, et l'entendement est dépassé. Dans le même sens, Kant évoque plus loin «une représentation de l'imagination qui appartient à sa présentation [la présentation d'un concept], mais qui, par elle-même, fournit l'occasion de penser bien davantage que ce qui se peut jamais comprendre dans un concept déterminé, et par conséquent élargit (erweitert) de manière esthétique le concept lui-même de manière illimitée "; une telle représentation relève de l'imagination créatrice, laquelle "met en mouvement le pouvoir des idées intellectuelles (la raison) et cela d'une manière qui lui permet, à propos d'une représentation, de penser bien plus [...] que ce qui en elle peut être appréhendé et rendu clair ${ }^{28} »$.

Tout converge, dans la description des procédés de l'imagination créatrice, vers ceci qu'il y va d'« un élan de l'imagination en vue de penser davantage, même si c'est de manière non explicitée, que ce qui se peut comprendre dans un concept, et par conséquent dans une expression linguistique déterminée ${ }^{29}$ ». L'idée esthétique "permet de penser [...] beaucoup d'indicible ${ }^{30} »$ : ce champ de la pensée ouvert, investi et animé de la sorte par l'imagination créatrice, et s'étendant «à perte de vue ${ }^{31}$ ", comporte de l'indicible en droit. C'est en quoi l'idée esthétique n'est jamais que suggestive, sa richesse demeure dans l'implicite; elle n'offre en aucune manière une mise en présence - sous les yeux - de quelque chose qui pourrait être dit par ailleurs : le génie ne pourra pas expliquer sa création; l'imagination dit «à sa manière » tout ce qu'il y a à dire, et ne peut l'être autrement.

On est loin de Batteux qui indiquait que l'art ne fait que perfectionner les expressions de la nature (qui se comprennent « naturellement ${ }^{32} »$ ).

L'idée esthétique, loin d'avoir son site originel au plus près de la perception sensorielle, est bien plus apparentée aux idées intellectuelles. Bien sûr ces idées esthétiques sont 
des "intuitions", mais des intuitions "intérieures" auxquelles "aucun concept ne peut être complètement adéquat $»^{33}$. L'idée esthétique est une instance sensible (non rationnelle ou intellectuelle) mais porteuse de sens, un sens qui ne saurait être dit en langage conceptuel, cognitif. C'est une intuition faite sens, un sens fait intuition.

Le classement et la hiérarchie des arts qui suivent l'exposé sur le génie plaident, sans référence à une quelconque imitation, pour un statut où n'entre aucune composante de "ressemblance» dans l'activité de l'imagination créatrice d'idées esthétiques. La hiérarchie se fonde sur l'animation des facultés suscitée par l'expression de telles idées à même l'œuvre.

\section{Division des arts et médiums}

L'expression d'idées esthétiques est le point de départ de la division des beaux-arts. En effet, parce que l'objet produit par les beaux-arts est expression, on est justifié à classer ces arts selon une " analogie [...] avec le mode d'expression dont se servent les êtres humains quand ils parlent, afin de communiquer entre eux aussi parfaitement que possible, non seulement selon leurs concepts, mais aussi à travers ce qu'ils éprouvent ${ }^{34}$ ». On peut donc, non sans avoir indiqué que l'« esquisse " qui suit n'est pas à considérer comme "une théorie achevée ", et qu'il s'agit "d'une des multiples tentatives possibles que l'on peut et doit encore effectuer ${ }^{35}$ ", rappeler, aux desseins de cette analogie, que le mode d'expression langagier est en fait composé d'une triade constituée « du mot, du geste et du ton (articulation, gesticulation et modulation) ».

C'est, explique Kant, uniquement la combinaison de ces trois sortes d'expression qui définit la communication complète dont s'acquitte le locuteur. Car la pensée, l'intuition et la sensation se trouvent ainsi, en même temps et de manière unifiée, transmises à d'autres.

21 Par analogie, dès lors, il n'y a " que trois espèces de beaux-arts : l'art de la parole, l'art plastique (die bildenden Künste ${ }^{36}$ ) et l'art du jeu des sensations (en tant qu'impressions externes des sens) ».

Sans prétendre que ce classement des beaux-arts par Kant présente une très grande originalité ou qu'il soit sans précédent ${ }^{37}$, il s'avère significatif pour le présent propos de relever ce que son principe met en exergue autrement que chez les critiques dont les noms sont évoqués, et qui concerne la portée des différences entre médiums artistiques. Pour limiter l'entreprise aux dimensions ici imparties, le foyer de l'analyse sera constitué par les éléments essentiels relatifs à la poésie et à la peinture ${ }^{38}$.

Si le mode d'expression qu'est la parole sert de référence avec ses trois éléments constitutifs (mot, geste, ton), ceux-ci renvoient, à l'évidence, aux analyses de l'usage du langage et aux traités de rhétorique qui servaient classiquement de repères aux théoriciens des arts, et de la peinture en particulier.

Il convient de noter que si le mot (l'énonciation ou l'élocution) est d'emblée attaché à la pensée, le geste l'est à l'intuition, c'est-à-dire à l'expérience perceptive, et le ton à la sensation (la donnée sensorielle). Plus élémentaire que l'intuition, la sensation est plus immédiatement porteuse d'affects: émotions, sentiments ${ }^{39}$. L'analogie avec ces éléments constitutifs du langage détermine dès lors trois classes de beaux-arts.

La classification se fait par la différence des médiums si l'on veut bien entendre par là des moyens d'expression d'idées esthétiques, ces moyens ou véhicules étant toujours des 
formes. En s'en tenant d'assez près à l'usage, Kant place dans la première classe (les arts de la parole ou du mot) l'éloquence et la poésie; dans la seconde (les arts du geste), la plastique et la peinture ; enfin dans la troisième (les arts du ton), la musique et l'art des couleurs.

Dans les arts du mot, l'opposition entre l'éloquence et la poésie annonce déjà largement le paragraphe sur la hiérarchie entre les arts, en soulignant que l'orateur manque à sa promesse d'une tâche conceptuelle, en vue d'une fin déterminée, en ce sens qu'il y introduit « un jeu divertissant de l'imagination » (ce qui n'est pas digne du sérieux de la tâche annoncée), tandis que le poète, n'annonçant qu'un jeu avec des idées, «accomplit quelque chose qui est digne d'une tâche ", car son jeu alimente l'entendement et « donne vie à ses concepts par l'intermédiaire de l'imagination ».

véritél'apparenceLaocoon

Dans la plastique (au sens plus strict), la sculpture est définie comme "l'art qui présente physiquement les concepts de certaines choses telles qu'elles pourraient exister dans la nature (cependant, en tant qu'elle constitue un des beaux-arts, en tenant compte de la finalité esthétique) ", c'est-à-dire sans assujettissement au concept. Quant à l'architecture, il est clair en revanche que sa recherche ne saurait être la seule expression d'idées esthétiques, puisque c'est « un certain usage de l'objet artistique qui constitue le point essentiel», ce qui vient "limiter» les idées esthétiques ${ }^{40}$. La sculpture, de son côté, a l'expression des idées esthétiques comme "but principal ». C'est dire que Kant, sans rejeter tout à fait le vocabulaire de l'imitation, prend toujours garde de restreindre la portée de cette façon de parler : une œuvre figurative est « faite uniquement pour être contemplée ${ }^{41}$ ", elle "doit plaire par elle-même"; si elle est donc en un sens " une simple imitation de la nature ", c'est en un sens qui "se réfère à des idées esthétiques ", leur expression étant l'essentiel, ce qui veut dire en un sens qui dès lors comporte cette restriction intrinsèque de l'imitation déjà évoquée : l'œuvre ne peut cesser d'apparaitre comme art.

Par ailleurs, et c'est un second point intéressant quant à ces arts du geste, la peinture reçoit elle aussi une subdivision (laquelle ne nous est pas familière), en tant qu'art qui "présente l'apparence sensible artistiquement liée avec des idées", puisqu'elle comprend d'une part les " arts de la belle description ${ }^{42}$ de la nature » et d'autre part les " arts du bel agencement de ses produits "; ce qu'il faut entendre comme la peinture proprement dite d'un côté: Malerei, et l'art des jardins de l'autre. La peinture proprement dite ne produit que "l'apparence de l'extension physique ${ }^{43}$ ", le jardin a une extension réelle mais « ne fournit que l'apparence de l'utilisation et de l'usage pour d'autres fins que celles du simple jeu de l'imagination dans la contemplation de ses formes ». Kant éprouve le besoin d'expliquer dans une note cette présence "étrange " de l'art des jardins comme espèce du genre pictural. Nous avons bien, rassemblés ici, deux arts de l'espace ou de l'extension, selon la distinction chère à Lessing. Mais l'agencement artistique des formes naturelles elles-mêmes est tout autre que celui de la nature et il est "adéquat à certaines idées", de sorte que, comme le produit de la peinture proprement dite, il «n'est lui aussi destiné qu'à la vue », et l'on retrouve de la sorte également la distinction des arts selon le sens auquel chacun d'eux s'adresse (comme chez Batteux). C'est donc du point de vue de cette seule destination à la contemplation qu'il faut distinguer le classement de l'architecture et celui de l'art des jardins : la première est comme une sculpture subordonnée à un concept, le second est comme une peinture qui se joue dans l'espace de la nature réelle, mais qui, comme la 
peinture proprement dite, vise exclusivement au « libre jeu de l'imagination dans la contemplation ».

Dans cette ligne, la peinture est élargie encore à tout ce qui a trait à la décoration et à l'ameublement, jusqu'à l'art de s'habiller, tant il est vrai qu'à l'occasion d'une fête "pleine d'éclat ", explique Kant, tous ces éléments forment " une sorte de tableau qui, tout comme les tableaux proprement dits (qui n'ont pas pour intention d'enseigner par exemple l'histoire ou la connaissance de la nature), n'existe que pour être vu et afin de soutenir l'imagination dans son libre jeu avec des idées et d'occuper sans fin déterminée la faculté de juger esthétique ${ }^{44} »$. La parenthèse vaut dans cette citation son pesant d'or, car si l'art des jardins se trouve valorisé comme peinture, la peinture didactique, à l'inverse, est rabaissée au moins au rang de l'architecture qui ne peut être purement et simplement un bel art, trop liée qu'elle est à des fins déterminées et à une utilité qui lui est essentielle. Les arts "décoratifs" (au sens que l'on vient d'évoquer) sont quant à eux valorisés comme beaux-arts dans la mesure où l'on y peut juger des formes sans considération d'une fin, en fonction de l'effet produit sur l'imagination. Peu importe donc aux yeux de Kant le caractère très mécanique de certains de ces arts, et le caractère techniquement fort différent des uns et des autres. Le médium commun à tous ces arts est constitué par les formes offertes dans l'espace à la vue en tant qu'exprimant des idées esthétiques, c'est-à-dire analogiquement seulement évoquant des idées, au sens kantien strict cette fois. Tel est le médium. Selon quoi cette peinture élargie chez Kant est bien moins limitée au visible (elle renvoie à l'invisible) que celle que Lessing oppose à la poésie, et bien moins limitée à l'imitation de formes naturelles visibles ${ }^{45}$.

Enfin, Kant n'omet pas de dire en quoi ces arts des figures peuvent être analogues aux gestes, sont des arts du geste, par-delà l'analogie évidente qui veut que le geste accompagnant la parole soit la part de la communication qui est saisie par la vue et qui déploie une dimension spatiale : «[...] l'esprit de l'artiste, à travers ses figures, donne une expression physique de ce qu'il a pensé et de la façon dont il l'a pensé, et [...] il fait parler la chose elle-même, pour ainsi dire par une mimique: c'est là un jeu très habituel de notre imagination qui suppose une âme (Geist) aux objets inanimés d'après leur forme. " L'accent est mis à nouveau sur l'animation de l'imagination et sur les idées esthétiques ${ }^{46}$. En outre, Kant insiste sur le fait que ce n'est que par analogie que les arts dont il vient de parler se rapportent au rôle du geste dans la communication verbale. C'est dire qu'il n'y a dans ces arts qu'une similitude au rapport qui est celui du geste à la parole. Autrement dit, la similitude se trouve entre le rapport des gestes du locuteur à sa parole (qui elle est porteuse de sens définis par des concepts déterminés), d'une part, et, d'autre part, le rapport des formes et configurations de la peinture au sens indéterminé vers lequel font signe les idées esthétiques qu'elles véhiculent ; avec ce résultat sans doute que le rapport animé des facultés que sont l'imagination et l'entendement dans un jeu libre suscité par la peinture est similaire au rapport de l'imagination à l'entendement correspondant au geste accompagnant la parole ; si c'est de sens qu'il s'agit dans les deux cas, aucun concept déterminé n'est en cause dans le bel-art. Quand on a affaire à un orateur, le sens de ses gestes ne peut être déterminé exactement que par référence à la parole (aux concepts qu'exprime la langue) qu'ils accompagnent : en lui-même le geste est porteur d'un sens qui n'est précisé que par les mots qu'il accompagne (sans eux son sens reste indéterminé).

31 Il est clair que, par-delà l'analogie des trois classes d'art à la triade parole, geste, ton, on s'écarte ici très largement d'un classement par matériaux, ou par types de techniques 
requises: il y a loin de l'art du couturier à celui des jardins. Le médium, on peut l'appeler ainsi car il s'agit bien du moyen ou de la médiation de la communication universelle à opérer, le médium qui les rassemble est la forme, une forme vivifiée par un sens, une forme avec ce que le terme comporte (dans ce cas-ci) de spatialité et de rapport à la vue, mais avec la nécessité de donner à penser, d'animer les facultés et de transmettre des idées esthétiques (et non d'imiter). On est loin également ici des différenciations par la matière première.

Il y a bien "transcendance du matériau », et la logique des regroupements effectués par Kant peut sembler intéressante aujourd'hui, car cet élargissement de l'art de peinture pourrait être considéré comme annonçant, ou anticipant, en un sens l'extension moderne de ce que nous appelons, encore, les arts plastiques: Land Art, Body Art, performance même à certains égards - à ce sujet on relèvera que l'art des jardins (pourtant catalogué art pictural) exige aussi une prise en considération du temps ${ }^{47}$. Du reste, il faut aussi rapprocher la danse (avec sa dimension temporelle intrinsèque) de cette catégorie des arts du geste ${ }^{48}$. On s'éloigne nettement de la séparation tranchée de Lessing.

Mais une autre considération complète ces remarques sur les arts plastiques : si d'un côté la peinture s'est élargie, elle se trouve, d'un autre, privée de ce que certains comptent comme son médium essentiel : la couleur ou le coloris. C'est que l'art des couleurs se trouve placé par Kant dans la troisième catégorie, celle qui correspond au ton, ce qui signifie pour lui qu'il s'agit d'un jeu des sensations.

Dans «l'art du beau jeu des sensations", une part ne peut être l'objet d'une communication universelle (en tout cas on ne peut le garantir), ce sont les sensations "qui sont produites de l'extérieur", c'est-à-dire qu'il s'agit d'un effet sur le sens interne qui est subi par l'individu et est tel que nous ne pouvons guère nous assurer de la manière dont un autre voit telles couleurs, dont il entend tels sons ${ }^{49}$; mais «le jeu [de ces sensations] doit cependant se pouvoir communiquer universellement»; sans quoi il n'y aurait ni beauté ni bel-art. Cet art ne peut pour ces raisons « concerner que la proportion des différents degrés de la disposition (tension) du sens dont relève la sensation, c'est-à-dire la tonalité de ce sens ». Autrement dit, une couleur seule, prise isolément, est difficilement belle à proprement parler, elle n'est le plus souvent guère autre chose qu'agréable ${ }^{50}$. C'est le jeu des sensations qui est plaisant, qui anime l'imagination et l'entendement de concert, c'est lui qui produit une forme. On peut alors avoir affaire à un jeu artistique des sensations de l'ouïe dans le temps (dans la musique) ou à un jeu artistique des sensations de la vue dans l'espace (dans l'art des couleurs).

Sans entrer dans les considérations de science physique qui sont évoquées à cet endroit, si on admet que l'on n'a pas affaire seulement à des impressions sensorielles mais bien à " un effet d'un jugement d'appréciation porté sur la forme susceptible d'être appréhendée dans le jeu des sensations multiples ", alors il s'agit bien de beauxarts et non de simple art d'agrément (comme dans la réalisation d'un mets délicieux au palais). On peut regretter que Kant ne prenne la peine ni de préciser cette situation qu'il donne au jeu des couleurs, par analogie avec le jeu des sons dans la musique, ni de commenter plus avant cette dissociation de ce qui constitue classiquement deux parties de l'art de peinture, le dessin et le coloris. C'est en tout cas une autre façon d'insister sur la nature commune des beaux-arts: ce sont les arts de l'expression des idées esthétiques, et les techniques ne sont pas des critères essentiels de classement, pas 
davantage que la référence à un sens plutôt qu'à un autre. Ce qui importe est bien pour Kant la manière d'animer les facultés, de faire jouer imagination et entendement : les moyens sont soit des figures, soit des agencements, des configurations de sensations dans le temps ou dans l'espace, relevant de l'ouïe ou de la vue. En tout état de cause, la séparation tranchée de Lessing entre des arts de l'espace et des arts du temps est, une fois de plus, mise à mal par cette troisième classe d'arts, comme l'est aussi la division plus classique par techniques ou organes des sens.

On retiendra donc cette conclusion qui est exprimée à propos de l'association des beaux-arts en un seul et même produit :

[...] dans tous les beaux-arts, l'essentiel réside dans la forme, laquelle, vis-à-vis de l'observation et du jugement d'appréciation, contient en elle une dimension de finalité, et où le plaisir est en même temps culture et dispose l'esprit à des idées en le rendant par conséquent capable d'éprouver bien davantage de plaisir et de divertissement de ce type ; l'essentiel ne réside pas dans la matière de la sensation (l'attrait ou l'émotion), où il s'agit uniquement de jouissance, laquelle n'apporte rien à l'idée, émousse l'esprit [...] ${ }^{51}$.

Animer l'imagination et l'entendement de concert par des idées esthétiques, c'est donc contribuer à cultiver l'esprit : le disposer à trouver toujours plus de satisfaction de ce type, et ainsi le disposer aux idées proprement dites, rationnelles.

\section{Hiérarchie et médiums actuels}

Après la présentation de ce classement, on ne peut plus s'étonner de la hiérarchie proposée, et qui n'est guère originale en elle-même. Cette hiérarchie est guidée d'une part par la priorité donnée au jeu libre des facultés, ce qui signifie qu'une liberté plus grande de l'imagination donne la priorité à la poésie en un sens grosso modo déjà présent chez Lessing.

Si Kant souligne ainsi que la poésie doit son premier rang à sa liberté presque totale et à sa capacité d'élargir l'esprit et à s'élever jusqu'aux idées par la réflexion, il écarte pour cause de tromperie ou de supercherie l'éloquence comme art de persuader, c'est-à-dire, précise-t-il, comme art « d'abuser par la beauté de l'apparence, et non pas l'art de bien parler » : la poésie l'emporte encore une fois en loyauté et sincérité ${ }^{52}$. Ensuite viendrait la musique à condition que l'on considère l'attrait et le mouvement de l'esprit, car alors la musique est l'art qui se rapproche le plus de la poésie et qui s'y associe naturellement, même si elle reste bien en deçà de celle-ci, puisque, contrairement à la poésie, elle ne laisse rien "derrière elle " pour la réflexion. Mais on regrette qu'elle soit davantage jouissance (plaisir sensoriel) que culture (élargissement de l'esprit). C'est pourquoi si l'on veut, en revanche, apprécier "la valeur des beaux-arts d'après la culture qu'ils procurent à l'esprit ${ }^{53}$ ", c'est la dernière place qui revient à la musique précisément en tant qu'elle charme plus qu'elle ne donne à penser. Il est intéressant de noter que Kant ne prend pas réellement la peine de situer les arts plastiques par rapport aux arts de la parole comme il vient de le faire pour la musique. Il se contente d'indiquer que dans cette dernière catégorie, il donne la préférence à la peinture : «en partie parce que, comme art du dessin, elle se trouve au principe de tous les autres arts plastiques; en partie parce qu'elle peut pénétrer largement plus avant dans la région des idées, et aussi élargir davantage, en conformité avec celles-ci, le champ de l'intuition ». Mais de ce que Kant a dit de la musique il convient de conclure que du point de vue le plus strict des beaux-arts, donc de l'animation des facultés plutôt que de l'attrait et des émotions, 
tous les arts plastiques derrière la peinture prennent le pas sur la musique dans le classement; derrière la peinture, et en plaçant très certainement l'architecture derrière la sculpture en raison de son lien à une utilité déterminée.

Ce que l'on peut retenir de cette approche, c'est qu'à l'encontre de Batteux, Kant propose une vue transversale par rapport aux différents sens auxquels les arts peuvent faire appel ; à l'encontre de Lessing, il brouille la distinction entre les arts de l'espace et les arts du temps; et contre la tradition, il met à mal une classification en termes de métiers ou de techniques mises en œuvre. Ce qui est commun aux arts, c'est que le sens l'emporte, un sens sensible (c'est la portée de l'expression d'idées esthétiques), mais un sens qui pointe par analogie et non pas par ressemblance ou par imitation vers du hors espace-temps, ou vers du non-audible, du non-visible. Le concept reste indéterminé, c'est le jeu des facultés qui l'emporte, donnant librement à penser de manière élargie. Le médium artistique est pour chaque art dans la forme, non pas en tant qu'elle peut être subsumée sous un concept déterminé, mais en tant qu'elle véhicule des idées esthétiques, c'est-à-dire une pensée libre et qui dépasse le monde sensible. Ce qui semble fournir une ouverture à une multitude quasi infinie de supports possibles de formes, ou de jeux de l'imagination et de l'entendement, puisque les formes et le jeu peuvent concerner la pensée, l'intuition ou la sensation, et bien entendu toutes sortes d'association entre ces dernières.

41 Cette ouverture possible à partir du classement et de la hiérarchie des beaux-arts chez Kant ne semble guère avoir été exploitée par les générations qui le suivirent immédiatement et s'inspirèrent pourtant largement de ses textes et en particulier de cette troisième Critique. La réflexion d'aujourd'hui pourrait avoir intérêt à y faire retour, pour aborder une gamme infinie de médiums qui dans le vocabulaire de Kant ne sont jamais néanmoins, dans leur infinie diversité, que formes et jeux, insistant plus ou moins sur les composantes de pensée, d'intuitions ou de sensations. La forme n'est nullement chez lui, "dessin» en un sens restrictif, elle est véhicule universel de significations, mais non de messages conceptuels. Le classement et la hiérarchie de Kant ne réduisent les arts (aucun d'eux) ou leurs médiums ni aux sens, aux impressions sensorielles, ni aux affects (individuels et impartageables, eux aussi), ni à l'intellectualité universelle du concept. Les médiums artistiques sont tout ce qui permet un jeu de l'intelligence et de la faculté sensible, et donne à penser (à se mouvoir dans un champ illimité de significations) et non à connaître. En insistant sur le partage universel, dans l'esprit des Lumières, il insiste beaucoup moins que ne le feront les romantiques sur une intériorité passionnée et émotive, et beaucoup moins que ne le fera l'Idéalisme allemand, chez un Hegel, sur le contenu rationnel, qui n'est chez ce dernier rien moins que l'Esprit absolu qui se dira finalement de manière satisfaisante seulement dans le savoir philosophique et par rapport auquel, par sa nature sensible, l'art demeurera toujours en défaut. L'Art au singulier n'est pas encore à l'ordre du jour chez Kant (mais bien l'autonomie de la sphère esthético-artistique). Sa pensée pourrait ainsi nourrir une réflexion soucieuse du respect des différences entre les médiums artistiques actuellement disponibles.

De sensibilité, Kant n'est (pour autant qu'on puisse donner un sens même très général à ces catégories) ni classique (il trouve la régularité vite pourvoyeuse d'ennui), ni romantique (la sensibilité exacerbée, l'expression des états d'âme individuels ne sont pas son affaire, pas plus que la passion pour un passé national). S'il a pu être jugé proche des romantiques, c'est dans son insistance sur la richesse d'imagination du 
génie, mais il met aussi anticipativement en garde contre tout débordement de cet ordre: le non-sens guette et doit être évité. Il reste attaché à l'appel à la raison universelle, et c'est ce qui fait sa parenté avec le classicisme. Il est un Aufklärer cosmopolitique, se réclamant de cet esprit des Lumières que les romantiques voudront balayer avec le classicisme. Hegel sera, de son côté, plus en phase avec le refus romantique des limites. Si Kant peut nous parler aujourd'hui, peut-être est-ce parce c'est d'une pensée des limites que nous avons le plus besoin aujourd'hui, de nos limites, en arts comme ailleurs. Cette pensée des limites libère bel et bien en un sens tous les médiums possibles.

\section{BIBLIOGRAPHIE}

Batteux Charles, Les Beaux-Arts réduits à un même principe, Édition critique par Jean-Rémy Mantion, Paris, Aux amateurs de livres, coll. « Théorie et critique à l'âge classique », 1989.

Kant Immanuel, Critique de la faculté de juger, traduction par Alain Renaut, Paris, GF Flammarion (Aubier), 1995.

Krajewski Pascal, «Qu'appelle-t-on un médium ? », dans Appareil [en ligne], 11/02/2015, consulté le 29 octobre 2015. URL : http://appareil.revues.org/2152.

Kristeller Paul Oskar, Le système moderne des arts. Étude d'histoire de l'esthétique, traduit de l'anglais par Béatrice Han, Nîmes, Jacqueline Chambon, coll. Rayon Art, 1999.

Lories Danielle, «Du cadre et de l'esthétique: Kant ou Derrida », in Lenain Thierry, Steinmetz Rudy (dir.), Cadre, seuil, limite, Bruxelles, La lettre volée, 2010.

\section{NOTES}

1. Pascal Krajewski, «Qu'appelle-t-on un médium? », Appareil [En ligne], Articles, mis en ligne le 11 février 2015, consulté le 11 juillet 2016. URL : http://appareil.revues.org/2152.

2. Qu'on peut écrire avec une majuscule pour mettre à distance tout ce à quoi renvoient encore, par-delà les « beaux-arts », les traductions modernes de technè ou ars.

3. Voir l'éditorial de ce numéro : https://appareil.revues.org/2281

4. Nous citons la traduction réalisée par A. Renaut de la Critique de la faculté de juger, désormais CFJ, Paris, GF Flammarion (Aubier), 1995, en l'amendant (rarement) et/ou en la complétant des termes allemands pour des questions de clarté, et ce, sans autre avertissement que la présente note. Nous indiquons le paragraphe et si c'est utile la page du tome $\mathrm{V}$ de l'édition de l'Académie. Ici : CFJ, § 51, p. 320.

5. CFJ, § 33, p. 284.

6. Paul Oskar kristeller, Le Système moderne des arts. Étude d'histoire de l'esthétique, Nîmes, Jacqueline Chambon, 1999.

7. Charles Batteux, Les Beaux-Arts réduits à un même principe, Paris, Aux amateurs de livres, 1989.

8. Ce qui lui vaudra rapidement de vives critiques, dont celle de Diderot. 
9. C. Batteux, Les Beaux-Arts réduits à un même principe, op. cit., p. 82. Il est ici question des beauxarts «par excellence », et il faut noter ce «par excellence » qui rend la liste qui suit non exclusive «tels sont la musique, la poésie, la peinture, la sculpture, et l'art du geste ou la danse ».

10. Ibid.

11. P. O. Kristeller, Le système moderne des arts, op. cit., p. 64, 66 .

12. On pourrait ajouter que les arts sont ici présentés dans un ordre hiérarchique, même si la contestation de cet ordre se trouve dans le texte même qui la présente. Les articles consacrés aux différents arts confirment la référence à l'imitation.

13. CFJ, § 45, p. 306.

14. Dans la seconde partie de son ouvrage, Batteux indique qu'«[il] établit le principe de l'imitation, par la nature et par les lois du goût "; il propose donc une définition du goût. C. Batteux, Les Beaux-Arts réduits à un même principe, op. cit., p. 113 et 115 .

15. CFJ, § 45, p. 306.

16. Ibid.

17. CFJ, § 49, p. 318.

18. «Ut pictura poesis » : la poésie comme la peinture ; la comparaison d'Horace fut très largement utilisée dans son inversion: la peinture comme la poésie, à partir de la Renaissance en particulier, et elle a sous cette forme permis de calquer les théories de la peinture sur la théorie antique de l'art des mots : aussi bien la rhétorique que la poétique, du reste. Dans le Laocoon, Lessing s'insurge contre cette réduction d'un art à l'autre, et accuse les différences.

19. Baumgarten utilise le terme et évoque le projet d'une nouvelle discipline scientifique dès 1735 ; le premier volume de son Aesthetica parait en 1750.

20. Cette parenté mériterait une étude serrée qui ne peut avoir sa place ici. Les comparaisons avec les deux références peuvent s'étendre à bien des notions : on pourrait aussi bien comparer l'imagination kantienne et celle mise en œuvre par Batteux. Et on pourrait voir dans cette simple phrase de Batteux toute la répartition des rôles que l'on trouve chez Kant: « Le génie et le goût ont le même objet dans les arts. L'un le crée, l'autre en juge. » (C. Batteux, Les Beaux-Arts réduits à un même principe, op. cit., p. 113) Chez Kant, voir en particulier CFJ, § 48 et $\S 50$.

21. CFJ, § 44, p. 304.

22. Dans le Laocoon, pourtant, il n'y a pas d'abandon de la terminologie de l'imitation, laquelle fera ailleurs l'objet de vives critiques de la part de Lessing (cf. sa Dramaturgie de Hambourg, 1767-69).

23. C. Batteux, Les Beaux-Arts réduits à un même principe, op. cit., p. 227 (nous soulignons).

24. On ne peut refaire ici l'histoire de la notion d'expression présente dès les débats de l'Académie parisienne: il s'agit alors seulement d'imitation, de représentation, et en ce sens même les moyens d'expression des arts sont empruntés à (imités de) la nature.

25. Il faut, en cas de conflit, dit Kant "[autoriser] qu'on lèse la liberté et la richesse de l'imagination, plutôt que de porter atteinte à l'entendement » (CFJ, § 50).

26. Dans la "Dialectique du jugement de goût ", Kant distingue deux manières de présenter (darstellen) un concept : l'une est connue, c'est le schème qui met en relation un concept avec l'objet d'intuition correspondant, dans le cadre cognitif. La seconde est indirecte : Kant a recours au langage de l'analogie pour parler de présentation symbolique. Ceci n'implique aucune ressemblance entre les termes: "entre un État despotique et un moulin à bras il n'y a assurément aucune ressemblance mais il $\mathrm{y}$ en a bel et bien une entre les règles de la réflexion sur eux et sur leur causalité » (CFJ, § 59, p. 352).

27. CFJ, § 49, p. 314 .

28. CFJ, § 49, p. 315.

29. Ibid.

30. Ibid.

31. Ibid. 
32. C. Batteux, Les Beaux-Arts réduits à un même principe, op. cit, p. 240 .

33. CFJ, § 49, p. 314.

34. CFJ $\S 51$, p. 320. Sans indication contraire toutes les citations de Kant sont désormais issues de ce $\$ 51$ dont le développement est suivi de près.

35. En note au § 51, p. 320, mise en garde réitérée en note, p. 323.

36. Il vaut mieux, pour les raisons que l'on va voir, dire ici « arts plastiques » que "figuratifs » comme disent certaines traductions. Kant inclut dans la catégorie l'architecture qui n'est pas un art de l'image à proprement parler, même si on pourrait dire qu'il s'agit d'un art de figures si on veut bien ôter de ce terme les connotations de copie ou de représentation. Et on ne peut guère parler d'art des formes car le beau en tout art est forme ou formation, chez Kant.

37. Voir chez Batteux l'ouverture du chap. 1 de la section $3^{\text {e }}$ (sur la musique et la danse) de la troisième partie (La vérification du principe d'imitation par son application aux différents arts) : le chapitre est intitulé « On doit connaître la nature de la musique et de la danse par celle des tons et des gestes", et commence ainsi : «Les hommes ont trois moyens pour exprimer leurs idées et leurs sentiments, la parole, le ton de la voix et le geste ». La parole est l'organe de la raison, le ton et le geste vont au cœur sans détour. L'exposé conclut que la musique et la danse sont imitations de la nature et plus spécialement des sentiments et passions (tandis que la poésie est imitation des actions), la distinction se retrouve bien sûr chez Lessing. C. Batteux, Les BeauxArts réduits à un même principe, op. cit., p. 231-233.

38. On reviendra brièvement sur le statut particulier de la musique à propos de la hiérarchie des arts, § 53.

39. Comme l'indique le statut de l'espace et du temps comme formes a priori de la sensibilité dans la première Critique, l'intuition a une dimension formelle que n'a pas la sensation, simple matière de la connaissance sensible.

40. Batteux imposait une règle aux arts à la fois utilitaires et faits pour le plaisir : ils ne méritent de rejoindre les autres beaux-arts qu'à condition de s'y soumettre : «dans les arts qui sont pour l'usage, l'agrément [doit prendre] le caractère de la nécessité même ", comme "dans les arts qui sont destinés au plaisir, l'utilité n'a droit d'y entrer, que quand elle est de caractère à procurer le même plaisir, que ce qui aurait pu être imaginé uniquement pour plaire » (C. Batteux, Les BeauxArts réduits à un même principe, op. cit., p. 240).

41. Il faut se souvenir que le jugement esthétique étant sans concept, la contemplation du beau ne saurait être faite d'une analyse qui s'efforcerait de vérifier la conformité du tableau ou de la statue à un concept ou à un récit quelconque.

42. Le terme allemand "Schilderung" renvoie bien plus à l'acte de peindre que notre "description»; «dépiction» éviterait mieux l'ambiguïté, si on le prend littéralement. L'allemand distingue beschreiben de schildern.

43. La sculpture qui (comme l'architecture) occupe les trois dimensions de l'espace est vérité en ce sens et non apparence.

44. Nous soulignons.

45. On comprend mieux dans cette perspective les exemples non figuratifs que donne Kant dans l'Analytique du beau (décor de papier peint, rinceaux et grecques, au § 16).

46. Ce sont elles que Kant a liées au Geist au début du $§ 49$ sur le génie.

47. Car la nature a ses saisons, et le spectacle changeant du jardin devrait viser à animer l'imagination en toute saison, sans même parler du simple temps de la promenade indispensable à apprécier un jardin.

48. Au § 52 Kant évoque l'association des beaux-arts, notamment au théâtre ; il est rappelé que la danse est "un jeu des figures». Le $§ 14$ avait donné la danse pour un jeu des figures "dans l'espace ", mais il est évident que ce jeu, du reste accompagné de musique, sur la scène, se déroule dans le temps, car comme le remarquait Batteux, la figure de la danse est vivante, en mouvement. 
49. $C f .$, CFJ, § 39 .

50. À ce sujet, $c f . \S 14$, où se trouvent déjà des possibilités qu'une forme soit offerte dans la perception d'une unique couleur à la réflexion (au jeu de l'imagination et de l'entendement), et non pas seulement un organe du sens mis en mouvement. Sur ce paragraphe cf. D. Lories, « Du cadre et de l'esthétique: Kant ou Derrida ", dans T. Lenain T., R. Steinmetz (dir.), Cadre, seuil, limite, Bruxelles, La lettre volée, 2010.

51. CFJ, § 52, p. 326, nous soulignons.

52. On ne peut donc pas soutenir que chez Kant les arts du langage l'emportent sur toute la ligne, seule la poésie l'emporte. L'éloquence est dévaluée par sa fin déterminée (et peu morale) (CFJ, $\S 53$, p. 326-327).

53. CFJ, §53, p. 329.

\section{RÉSUMÉS}

En se concentrant sur le classement et la hiérarchie des beaux-arts proposés par Kant, cet article s'efforce de situer ses thèses dans l'histoire du concept des beaux-arts au XVIII siècle. Comme ce concept peut être considéré comme un antécédent historique de la question du médium artistique, l'article montre en quoi les critères de classement et de hiérarchie des arts proposés par le penseur critique peuvent encore être pertinents pour réfléchir sur la grande multiplicité des médiums artistiques aujourd'hui.

INDEX

Mots-clés : beaux-arts, xviiie siècle, Kant, classification des arts, idées esthétiques, forme

\section{AUTEUR}

\section{DANIELLE LORIES}

Professeur de philosophie à l'université catholique de Louvain, B-1348 Louvain-la-Neuve, Belgique) danielle.lories@uclouvain.be 